Musées, Patrimoine et Culture scientifiques et techniques

$186 \mid 2019$

novembre-décembre 2019

\title{
Une définition inadaptée
}

CPMF

\section{OpenEdition}

\section{Journals}

Édition électronique

URL : http://journals.openedition.org/ocim/3386

DOI : 10.4000/ocim.3386

ISSN : 2108-646X

Éditeur

OCIM

Édition imprimée

Date de publication : 1 novembre 2019

Pagination : 18-20

ISSN : 0994-1908

Référence électronique

CPMF, "Une définition inadaptée », La Lettre de l'OCIM [En ligne], 186 | 2019, mis en ligne le 01 novembre 2020, consulté le 25 janvier 2021. URL : http://journals.openedition.org/ocim/3386 ; DOI : https://doi.org/10.4000/ocim.3386

Ce document a été généré automatiquement le 25 janvier 2021.

Tous droits réservés 


\section{Une définition inadaptée}

\section{CPMF}

\section{La Conférence Permanente des Muséums de France (CPMF)}

1 Les muséums d'Histoire naturelle sont les héritiers des cabinets d'Histoire naturelle des Lumières. Leurs collections sont constituées d'objets et d'ouvrages naturalistes et anthropologiques, fruits de la recherche scientifique et de la découverte de nouveaux mondes.

2 Aujourd'hui, la France compte 68 muséums en région en plus des 12 sites du Muséum national d'Histoire naturelle. Cela représente 86 millions de spécimens pour environ 7,4 millions de visiteurs en 2018.

3 La CPMF, qui regroupe aujourd'hui 44 muséums signataires de la charte portant une déontologie commune, s'est donnée pour missions :

4 - de fédérer les professionnels des muséums ;

5 - d'être le porte-parole des muséums auprès des instances publiques ou privées ;

6 - de créer des synergies et donner de la cohérence aux actions spécifiques des muséums ;

7 - de qualifier et porter la reconnaissance des cœurs de métiers propres aux muséums.

8 Ainsi, la publication de la nouvelle définition des musées par l'Icom en juillet dernier amène la CPMF à s'interroger sur son sens profond. Encore une fois, il est regrettable que les réflexions autour des musées ne prennent pas en compte la spécificité des muséums et ce, malgré le " poids » que pèsent leurs collections dans le paysage muséal et la résonance des thématiques abordées en regard des défis de notre humanité. La CPMF se devait donc de défendre une définition plus cohérente avec la réalité de nos établissements et de nos métiers via un document collectif recueillant l'adhésion de l'ensemble de ses membres. 


\section{Une définition floue et ambiguë}

9 À la lecture de cette définition, une première impression de complexité et de confusion découle du vocabulaire employé conduisant à des questionnements sémantiques multiples. L'emploi et la succession de mots polysémiques ouvrent ainsi la porte à des interprétations diverses selon le point de vue du lecteur dont l'issue inévitable est la mésinterprétation. Il semble également difficile pour les professionnels des musées de se reconnaître dans ces termes et de se les approprier. Le discours universaliste est poussé à son paroxysme, évoquant maladroitement des préoccupations déontologiques et humanistes redondantes et vaporeuses. Dès le début du texte, la « démocratisation inclusive et polyphonique " en est l'illustration parfaite. S'agit-il d'un musée où plusieurs voix s'expriment dans son discours muséographique? À quel niveau (des critères, théories, hypothèses, croyances)? Que deviennent les sciences, l'exposition des faits, de la recherche? L'objectif affiché d'aboutir à des musées "participatifs et transparents » n'est-il pas un risque de les rendre illisibles et nébuleux?

\section{L'effacement de termes fondamentaux}

Au-delà de cette distraction sémantique, les membres de la CPMF s'alarment de la définition qui en résulte et de l'omission pure et simple de termes fondateurs.

En premier lieu, les collections ont disparu. Les musées sont des dépositaires d'artefacts et de spécimens. Les notions de service et de public, sans plus de distinction, sont gommées par une rédaction déjà mentionnée comme trop complexe. Même si les musées «sauvegardent (...) pour les générations futures» et sont "dépositaires (...) pour la société", la notion de service pour le public est totalement absente. Pourquoi substituer le mot public, très large et sans caractérisation à des termes comme "société", « peuples» et « communautés »?

En outre, même si l'on retrouve le cortège des missions d'un établissement muséal, ces dernières n'apparaissent qu'en seconde intention, dans le deuxième paragraphe, de manière passive, alors qu'elles sont le socle de ce qui constitue un musée. Enfin, l'action de conserver disparaît au profit de celle de préserver. Or, même si les définitions données par le dictionnaire sont similaires, la notion de conservation recouvre pour les professionnels des musées un sens beaucoup plus large. La conservation des collections comprend la compréhension des facteurs de détérioration des collections, une expertise en conservation préventive, des connaissances historiques et techniques des objets, une veille dynamique de recherche d'informations, etc. Ce qui est par conséquent bien plus complet et approprié que le verbe " préserver ».

\section{Les musées ne sont pas des instruments politiques}

Une phrase en particulier constitue le point d'achoppement principal du texte finissant de le décrédibiliser à nos yeux. Les musées travailleraient «avec et pour diverses communautés ». Une fois de plus, le terme de communauté est ambigu. Les musées scientifiques et les muséums plus particulièrement s'inscrivent activement dans la recherche scientifique via des projets de recherche, la collecte de terrain et l'étude scientifique de leurs collections. Ce fonctionnement concourt à la légitimité 
scientifique de l'établissement et à la justesse des informations exposées. Au moment où la sauvegarde de la biodiversité est une préoccupation majeure de notre société, les muséums constituent des interlocuteurs privilégiés des citoyens comme des chercheurs, en tant qu'acteurs de la sensibilisation environnementale, centres d'informations et de savoirs, lieux de partage et de rencontre.

Ils constituent pour le public des figures de référence dans leurs domaines de compétences sur un territoire donné. En effet, s'ils «travaillent en collaboration active » avec les communautés d'universitaires, d'amateurs et de savants... Ils ont le devoir de conserver une intégrité et une neutralité propres à leurs missions de service public. Les musées scientifiques ne peuvent être les miroirs d'une idéologie ou d'une culture, ils sont les narrateurs du « dialogue critique » conjugué au présent.

\section{Une définition qui n'englobe pas la diversité des musées?}

Tous ces commentaires amènent à s'interroger sur les typologies de musées auxquelles cette définition s'adresse. La définition nouvellement proposée, voulue universelle, semble au contraire ne s'adresser à aucun d'entre eux dans sa globalité.

16 Si les muséums et les musées d'archéologie peuvent s'identifier dans les termes d'artefacts et de spécimens, qu'en est-il par exemple des musées techniques ou industriels?

$17 \mathrm{Au}$ moment où les muséums questionnent leurs pratiques déontologiques de conservation des collections, doit-on sceller les critères avancés ici «de contribuer à la dignité humaine et à la justice sociale, à l'égalité mondiale et au bien-être planétaire "? On ne peut remettre en cause l'intérêt patrimonial et scientifique des écorchés de Fragonard, mais respectent-ils la dignité humaine? La collecte de spécimens vivants dans leur milieu naturel est nécessaire à la compréhension des bouleversements environnementaux et de la disparition des espèces mais contribue-t-elle totalement au bien-être planétaire?

18 Si les musées d'ethnologie peuvent se considérer comme polyphoniques ou travailler avec des communautés, qu'en est-il des musées scientifiques?

Enfin, si tous les musées doivent être inclusifs, peuvent-ils être tous participatifs et transparents? Qu'est-ce que cela signifie réellement? Une fois de plus, tout est une question de point de vue.

20 Notre préconisation est celle d'une réécriture complète du texte, en faveur d'une définition courte, au vocabulaire clair et sans ambiguïté, où les missions fondamentales des musées seront assez élémentaires et exhaustives pour englober le plus de typologies de musées. Les muséums se distinguent des autres musées selon les termes évoqués plus haut et, à ce titre, seront davantage soucieux de vouloir disposer d'un texte cohérent avec la réalité professionnelle de leurs métiers.

21 Il est plus que nécessaire que la démarche de réécriture soit maintenant participative et inclusive. 


\section{RÉSUMÉS}

Cet article est extrait du dossier «Vers une nouvelle définition du musée ? » dirigé par Ewa Maczek, directrice par intérim de l'Ocim. La définition du musée proposée depuis 2007 par l'Icom fait référence dans la sphère muséale mondiale. Si la volonté de faire évoluer cette définition fait sens, la proposition du Comité pour la Définition du musée, perspectives et potentiels (MDPP) de l'Icom a surpris bon nombre de professionnels et d'institutions, et tout particulièrement en France. La lettre de l'Ocim leur a ouvert ses pages pour qu'ils partagent leurs réactions.

\section{INDEX}

Mots-clés : politique du patrimoine, organisation internationale

\section{AUTEUR}

CPMF

Conférence permanente des muséums de France 